\author{
EESTI NSV TEADUSTE AKADEEMIA TOIMETISED. 27. KOIDE \\ FUOSIKA * MATEMAATIKA. 1978, NR. 4 \\ ИЗВЕСТИЯ АКАДЕМИИ НАУК ЭСТОНСКОИ ССР. ТОМ 27 \\ ФИЗИКА * МАТЕМАТИКА, 1978, № 4
}

Ю. ЛЕМБРА

\title{
ОБ ОПТИЧЕСКИХ СВОЙСТВАХ НЕОДНОРОДНОГО СЛОЯ С ЭКСПОНЕНЦИАЛЬНОЙ ЗАВИСИМОСТЬЮ ПОКАЗАТЕЛЯ ПРЕЛОМЛЕНИЯ
}

I. LEMBRA. EKSPONENTSIAALSELT MUUTUVA MURDUMISNÄITAJAGA MITTEHOMOGEENSE KIHI OPTILISTEST OMADUSTEST

I. LEMBRA. ON THE OPTICAL PROPERTIES OF INHOMOGENEOUS FILM WITH THE EXPONENTIAL LAW FOR REFRACTIVE INDEX

\section{(Представлена П. Кардом)}

В данном сообщении мы вычислим коэффициент отражения неоднородного оптического слоя, показатель преломления которого изменяется по экспоненциальному закону

$$
n(z)=A e^{B z / h},
$$

при наклонном падении света, поляризованного перпендикулярно плоскости падения, используя схему, предложенную в $\left[{ }^{1,2}\right]$. В формуле (1) $z$ обозначает координату внутри слоя вдоль нормали к слою, $A$ и $B$ - безразмерные вещественные постоянные, $h$ - толщина слоя.

Обозначив показатели преломления на границах слоя $z_{1}$ и $z_{2}$ через $n_{1}$ и $n_{2}$ соответственно, запишем формулу (1) в виде

$$
n(z)=n_{1}\left(n_{2} / n_{1}\right)^{\left(z-z_{1}\right) / h} .
$$

Если использовать понятие эффективного показателя преломления $\left[{ }^{1,3}\right]$, то волновое уравнение принимает в рассматриваемом случае вид

$$
d^{2} U / d z^{2}+k^{2}\left(A^{2} e^{2 B z / h}-C^{2}\right) U=0,
$$

где $U$ - световое возбуждение, $k-$ волновое число, а постоянную $C$ следует выбрать из формулы

$$
C=n_{1}^{0} \sin \vartheta_{1}^{0} .
$$

Здесь $\vartheta_{1}^{0}-$ угол падения и $n_{1}^{0}-$ показатель преломления ограничивающей среды со стороны падения света.

Волновое уравнение (3) подстановкой (см. $[4,5])$

$$
y=(\alpha A / B) e^{B z / h}
$$

( $\alpha=h k-$ безразмерная спектральная переменная) приводится к уравнению Бесселя $p$-го порядка 


$$
y^{2} d^{2} U / d y^{2}+y d U / d y+\left(y^{2}-p^{2}\right) U=0,
$$

где

$$
p=\alpha C /|B| \text {. }
$$

Поэтому линейно независимые решения волнового уравнения можно выбрать следующим образом:

$$
\begin{aligned}
& U_{1}(z)=J_{p}\left((\alpha A / B) e^{B z / h}\right), \\
& U_{2}(z)=N_{p}\left((\alpha A / B) e^{B z / h}\right),
\end{aligned}
$$

где $J_{p}$ и $N_{p}$ - функции Бесселя первого и второго рода $p$-го порядка. Далее используем метод матрицы интерференции $\left[{ }^{1}\right]$. Из решений (8) можно составить характерные для этого метода определители $\Delta, D_{1}$, $D_{2}, D$ и $D_{12}$. После этого получим элементы матрицы интерференции сразу по формулам (5) из $\left[{ }^{2}\right]$ :

$$
\begin{aligned}
L_{12,11}^{0}=L_{12,22}^{0^{*}}= & {\left[\pi \alpha\left(n_{1}^{0} n_{2}^{0} \cos \vartheta_{1}^{0} \cos \vartheta_{2}^{0}\right)^{1 / 2} / 4 \ln \left(n_{2} / n_{1}\right)\right] \times } \\
\times & {\left[\eta_{2}^{0} S_{2}-\eta_{1}^{0} S_{1}+i\left(S+\eta_{1}^{0} \eta_{2}^{0} S_{12}\right)\right], } \\
L_{12,21}^{0}=L_{12,12}^{0 *}= & {\left[\pi \alpha\left(n_{1}^{0} n_{2}^{0} \cos \vartheta_{1}^{0} \cos \vartheta_{2}^{0}\right)^{1 / 2} / 4 \ln \left(n_{2} / n_{1}\right)\right] \times } \\
\times & {\left[\eta_{1}^{0} S_{1}+\eta_{2}^{0} S_{2}+i\left(S-\eta_{1}^{0} \eta_{2}^{0} S_{12}\right)\right], }
\end{aligned}
$$

где у $L$ первые нижние индексы указывают на ограничивающие среды, а два последних обозначают строку и столбец соответственно.

Для величин $S_{1}, S_{2}, S$ и $S_{12}$ имеют место следующие формулы:

$$
\begin{aligned}
S_{1} & =J^{\prime}{ }_{p 1} N_{p 2}-J_{p_{2}} N_{p_{1}}^{\prime}, \\
S_{2} & =J_{p_{1}} N_{p_{2}}^{\prime}-J_{p_{2}}^{\prime} N_{p 1}, \\
S & =J_{p_{1}} N_{p_{2}}-J_{p_{2}} N_{p 1}, \\
S_{12} & =J_{p_{1}}^{\prime} N_{p_{2}}-J^{\prime}{ }_{p 2} N^{\prime}{ }_{p 1} .
\end{aligned}
$$

В формулах (10) штрих у функции Бесселя означает производную по $y$, а второй индекс указывает на аргумент $y_{1}$ нли $y_{2}$ соответственно; при этом

$$
y_{1}=\alpha n_{1} / \ln \left(n_{2} / n_{1}\right), \quad y_{2}=\alpha n_{2} / \ln \left(n_{2} / n_{1}\right) .
$$

Величины $\eta_{1}^{0}$ и $\eta_{2}^{0}$ определяются с учетом показателей преломления $n_{1}^{0}$ и $n_{2}^{0}$ ограничивающих сред:

$$
\eta_{1}^{0}=n_{1} / n_{1}^{0} \cos \vartheta_{1}^{0}, \quad \eta_{2}^{0}=n_{2} / n_{2}^{0} \cos \vartheta_{2}^{0},
$$

где $\vartheta_{2}^{0}-$ угол преломления в ограничивающей среде вблизи границы $z_{2}$.

Путем использования рекуррентных формул для функций Бесселя можно из (10) исключить производные:

$$
\begin{gathered}
S_{1}=\mu_{1} S+W_{1}, \\
S_{2}=\mu_{2} S+W_{2}, \\
S_{12}=\mu_{1} \mu_{2} S+\mu_{2} W_{1}+\mu_{1} W_{2}+W_{12},
\end{gathered}
$$

где

$$
\mu_{1}=\left(n_{1}^{0} \sin \vartheta_{1}^{0} / n_{1}\right) \operatorname{sgn} \ln \left(n_{2} / n_{1}\right),
$$




$$
\begin{gathered}
\mu_{2}=\left(n_{1}^{0} \sin \vartheta_{1}^{0} / n_{2}\right) \operatorname{sgn} \ln \left(n_{2} / n_{1}\right) \\
W_{1}=J_{p 2} N_{p+1,1}-J_{p+1,1} N_{p 2}, \\
W_{2}=J_{p+1,2} N_{p 1}-J_{p_{1}} N_{p+1,2}, \\
W_{12}=J_{p+1,1} N_{p+1,2}-J_{p+1,2} N_{p+1,1} .
\end{gathered}
$$

Здесь второй индекс у функции Бесселя по-прежнему указывает на аргумент $y_{1}$ или $y_{2}$ соответственно.

Из (9) для вычисления коэффициента отражения непосредственно вытекает следующая компактная формула:

$$
R_{12}=\frac{\left(\eta_{1}^{0} S_{1}+\eta_{2}^{0} S_{2}\right)^{2}+\left(S-\eta_{1}^{0} \eta_{2}^{0} S_{12}\right)^{2}}{\left(\eta_{1}^{0} S_{1}-\eta_{2}^{0} S_{2}\right)^{2}+\left(S+\eta_{1}^{0} \eta_{2}^{0} S_{12}\right)^{2}} \text {. }
$$

\section{Л И Т Е Р А Т У Р А}

1. Қ ард П. О матрице интерференции неоднородного оптического слоя. - Изв. АН ЭССР, Физ. Матем., 1974, т. 23, № 2, с. 113-119.

2. Л ембра Ю. Об использовании матрицы интерференции неоднородного оптического слоя. - Изв. АН ЭССР, Физ. Матем., 1975, т. 24, № 3, с. 341-342.

3. К ард П. Г. Анализ и синтез многослойных интерференционных пленок. Таллин, 1971.

4. Бреховских Л. М. Волны в слоистых средах. Изд. 2-е. М., 1973, с. 131.

5. К а мке Э. Справочник по обыкновенным дифференциальным уравнениям. Изд. 4-e. M., 1971, c. 402.

\section{Тартуский государственный} университет
Поступила в редакцию

$16 / \mathrm{I} \quad 1978$ 